\title{
Speciation and the evolution of gamete recognition genes: pattern and process
}

\author{
SR Palumbi \\ Department of Biology, Hopkins Marine Station, Stanford University, Pacific Grove, CA, USA
}

Proteins on gamete surfaces are major determinants of fertilization success, particularly in free-spawning animals. Molecular analyses of these simple genetic systems show rapid evolution, positive selection, accelerated coalescence and, sometimes, extensive polymorphism. Careful analysis of the behavior of sperm produced by males with different gamete alleles shows that these alleles can deliver significant functional differences. Three forms of allele-specific fertilization advantage have been shown: assortative mating based on gamete type, rare allele advantage and heterozygote superiority. Models suggest that sperm and egg proteins may be coevolutionary partners that can alternate between directional selection for high fertilization ability and cyclic adaptation of eggs and sperm driven by sexual conflict. These processes act within allopatric populations and may accelerate their divergence if gamete adaptations in separate demes reduce cross-fertilization. Reproductive character displacement by reinforcement may play a diversifying role when previously allopatric populations rejoin. In circumstance that might prove to be common, divergence in sympatry can be driven by sexual conflict or by association of mating types with ecological differences. The ecology of fertilization, especially the degree of sperm competition and egg death via polyspermy, are important determinants of the strength and direction of selection on gametes. Freespawning animals allow careful analysis of gamete recognition -from the behavior of adults and interactions of gametes, to molecular patterns of allele divergence. Future research efforts on the evolutionary consequences of fertilization ecology, and the interaction between extensive variation in egg surface proteins and sperm fertilization ability, are particularly needed.

Heredity (2009) 102, 66-76; doi:10.1038/hdy.2008.104; published online 19 November 2008

Keywords: bindin; lysin; sexual conflict; reinforcement; positive selection; fertilization; heterozygote advantage

'Two things that are certain in life are death and taxes.' (Benjamin Franklin 1789)

Before either death or taxes, generally comes fertilization, the interaction and union of two gametes. Fertilization occurs after gamete attachment and fusion, which depends on the action of binding-effector proteins and enzymes that act at cell surfaces. These mechanisms can be species specific or quite general among taxa (Vieira and Miller, 2006). Because basic fertilization demands the interaction of proteins produced by male and female genomes, it can be driven by evolutionary pressures that act on mating systems such as gender conflict (Gavrilets and Waxman, 2002; Gavrilets and Hayashi, 2005; Tomaiuolo et al., 2007), reinforcement (Geyer and Palumbi, 2003) or other aspects of the evolution of reproductive isolation (Tomaiuolo et al., 2007). Because gamete recognition involves relatively few gene products, as opposed to more complex mating barriers in fish and many terrestrial animals, the evolutionary pressures that act to cause reproductive isolation may

Correspondence: Professor SR Palumbi, Department of Biology, Stanford University, Hopkins Marine Station, 120 Ocean View Blvd, Pacific Grove, CA 93950, USA.

E-mail: spalumbi@stanford.edu

Received 4 March 2008; revised 27 July 2008; accepted 15 August 2008; published online 19 November 2008 be more obvious in these simple genetic systems (Palumbi, 1992).

Over the past 15 years, study of gamete recognition mechanisms have resulted in several broad generalizations about the patterns of gene evolution associated with rapid evolution of reproductive isolation. Here, I review basic fertilization patterns associated with gamete recognition including species-specific fertilization and conspecific sperm precedence. Patterns of evolution of gamete recognition genes that appear to apply across different taxa include rapid evolution of testes proteins, positive selection and repetitive amino-acid motifs of egg surface receptors. The level of intraspecific polymorphism, the intensity of selective sweeps within species and the speed of divergence between species vary among taxa in important ways. The types of mechanisms that have been proposed to generate these patterns include gender conflict sparked by polyspermy, heterozygote or rare allele advantage, and reinforcement driven by hybrid disadvantage in sympatric species. The ecology of fertilization plays a strong role in the intensity of these evolutionary forces, which can wax and wane during the history of a species. The end product is a constellation of genetic patterns that can play a strong role in species diversification across taxa.

Interest in gamete recognition evolution stems from observation of a number of features of fertilization systems. These features can be general across taxa, but have been best studied in free-spawning invertebrates or 
a number of well-investigated internally fertilizing insects or mammals. Detailed research has shown that union of gametes from two different species may be halted at many levels. Behaviors preventing or slowing mating between individuals of different species are well known, even in broadcast-spawning marine invertebrates considered to have simple mating systems (Lessios, 2007). Similarly, developmental breakdown in hybrid offspring, after fertilization, has been well described in some systems (for review see Coyne and Orr, 2004). Because reproductive isolation can occur at all these different points in time, fertilization failure has been described as a postmating, prezygotic barrier (Servedio and Noor, 2003).

When it occurs, species-specific fertilization due to gamete recognition can be recognized by failure of sperm (or pollen) from one species to fertilize eggs of another. In free-spawning animals or plants, these data have been generated by combining gametes in field or lab settings (Palumbi and Metz, 1991, for example). For internally fertilizing animals, gamete recognition failures can sometimes be inferred by low egg production or production of infertile eggs after interspecific insemination. However, there are many stages after insemination, but before fertilization, at which females can prevent interaction between eggs and sperm (Eberhard, 1996). As a result, ascribing fertilization failure to gamete recognition in these circumstances is difficult.

The two best-studied animal gamete recognition systems are fertilization in sea urchins and abalone. In sea urchins, sperm are activated by elements of the carbohydrate coat surrounding the eggs (called the jelly coat) and in some cases fertilization between species is inhibited by low activation (Biermann et al., 2004). In most cases, however, fertilization is inhibited by failure of the bindin protein on the sperm to attach to a sperm receptor on the egg (Metz and Palumbi, 1996). In abalone, a completely different system exists in which a protein called lysin on the sperm interacts with an egg protein named VERL to form a pore in the chorion surrounding the egg and allow sperm entry (Swanson and Vacquier, 1998).

In some species of the abalone genus Haliotis and the sea urchin genus Echinometra, species-specific gamete interactions control fertilization, allowing only conspecific sperm to enter eggs (Vacquier et al., 1990; Palumbi and Metz, 1991). In these cases, hybrids in the field are rare, despite the opportunity for mating (Palumbi and Metz, 1991). In a second set of cases, gamete interactions appear to generate only partial failure of fertilization (for example, in reef building corals, see Willis et al., 2006). In still other cases, barriers to fertilization are one-way: even though eggs of species A disallow fertilization by sperm of species B, A-sperm will fertilize B-eggs (Lessios, 2007).

For some species, partial or one-way barriers to fertilization might be an artifact of performing fertilization experiments with sperm of only one species at a time. For example, sperm of the sea urchin Echinometra oblonga can easily fertilize eggs of $E$. sp. $C$ when these eggs have no choice and vice versa. But when sperm of the two species are mixed, eggs of $E$. sp. $C$ are fertilized by conspecific sperm at much higher rates (Geyer and Palumbi, 2005). Such conspecific sperm precedence (Figure 1) is a common feature of internally fertilizing insects and mammals (Howard, 1999; Howard et al., 2008), but has rarely been tested for explicitly among broadcast spawners.

A recent review of reproductive isolation in broadcast spawners suggests that in many of the cases where gamete recognition is not strong enough to provide a perfect barrier to interspecific gene flow, ecological factors such as habitat segregation and reproductive timing often add additional opportunities for reproductive isolation (Lessios, 2007). The relative contribution of ecological and recognition barriers in such cases have seldom been quantified.

\section{Process and patterns of evolution at gamete recognition loci}

Conventional wisdom suggests that the fertilization system is so critical to reproductive success that

Fertilization between sea urchins species

No choice experiments

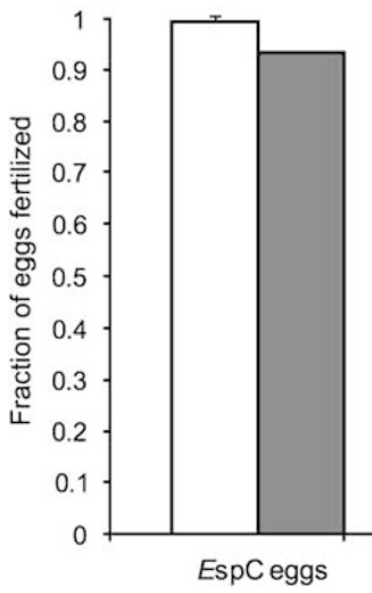

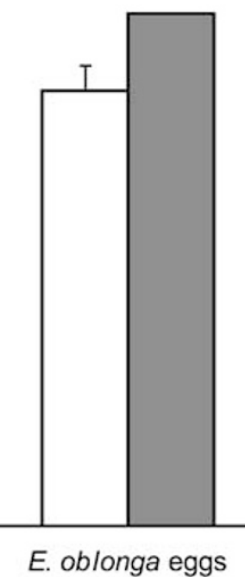

Eggs choosing among sperm

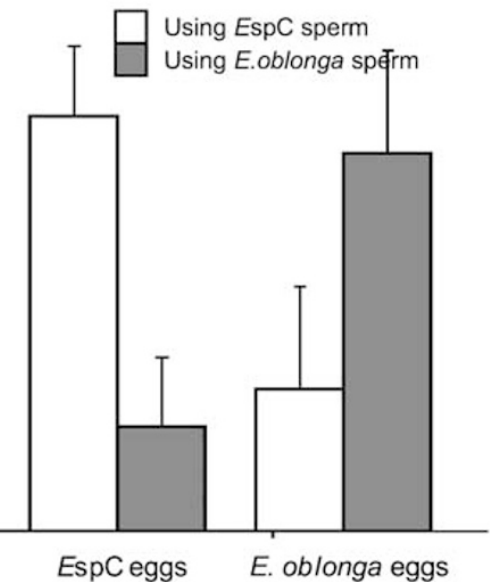

Figure 1 When eggs of the sea urchins Echinometra oblonga and E. sp. C. are given sperm from either species in no choice experiments, interspecific fertilization rate is high. However, when sperm from the two species are mixed in equal proportions, eggs are 2.5-4 times more likely to be fertilized by conspecific sperm, showing that conspecific sperm precedence is strong in these sympatric species (Geyer and Palumbi, 2005) 
evolution should tune it to be as efficient and flawless as possible. If this were the dominant evolutionary mechanism, then the reproductive system should evolve to the point where fertilization was mechanistically efficient, and then be under strong stabilizing selection to remain there, resulting in gamete recognition genes that were under purifying selection with few functional polymorphisms. In some cases, these expected patterns are seen. The large central stretch of the bindin gene-which is nearly identical in amino-acid sequences across most families of sea urchin-is perhaps an example of this process in action (Zigler et al., 2005). However evolutionary patterns of gamete recognition genes are often very divergent from this, and these genes can be some of the most quickly evolving genes in a genome. In particular, rapid divergence between species by positive selection is a common feature of gamete recognition proteins.

\section{Positive selection and rapid divergence}

One of the strongest signals of positive selection in any protein occurs in the lysin gene of abalone (Lee et al., 1995; Swanson and Vacquier, 2002a, b). In comparisons of this protein, amino-acid replacements occur much more commonly than silent substitutions, calculated on a siteby-site basis. Such a signal, called a high Dn/Ds ratio has been generally taken as a signal of natural selection for amino-acid variation, and can be seen between or within species (Hughes and Nei, 1988, 1989). Positive selection generates rapid divergence of proteins at the amino-acid level, and probably results in more rapid functional differentiation of gamete proteins in diverging species. Lysins in mussels and turban snails also evolve rapidly with high Dn/Ds values (Hellberg et al., 2000; Riginos et al., 2006).

In sea urchins, molecular evolution of the protein bindin, which attaches sperm to eggs, also occurs through positive selection, primarily in the first exon of the functional gene region (Metz and Palumbi, 1996) as well as through insertion and deletion of repetitive amino-acid motifs in exons one and two (Biermann, 1998; Debenham et al., 2000). However, these patterns do not occur among all sea urchin species, and seem to be strongest in genera that show large numbers of sympatric species pairs (Metz et al., 1998; Palumbi and Lessios, 2005; Lessios, 2007).

Across taxa, other genes expressed during reproduction, in gametes or gonads or reproductive tracks, tend to have high evolutionary rates and high values of Dn/Ds. The Accessory Proteins produced by male Drosophila show a high value of Dn/Ds, suggesting rapid evolution of these reproductive proteins (Panhuis et al., 2006). Twodimensional gel electrophoresis of proteins in gonad tissues show a higher level of differentiation than proteins from other adult tissues, suggesting particularly rapid evolution in testes (Thomas and Singh, 1992). Likewise, comparison of genes expressed during reproduction in primates show higher Dn/Ds than other tissues (Wyckoff et al., 2001). In a careful study of the rate of amino-acid evolution in genes expressed during different phases of development, Davis et al. (2005) found particularly high rates of amino-acid evolution of genes expressed in adult males.

Positive selection in egg proteins that bind sperm is also known for the VERL gene in abalone (Galindo et al.,
2003) and among repeated amino-acid motifs in the EBR1 protein in sea urchins. In addition, positive selection has also been found in the mammalian zona pellucida proteins ZP2 and ZP3 (Swanson et al., 2001; Turner and Hoekstra, 2006). Interpretation of the ZP protein results is complicated by elegant experiments in which the human versions of ZP2 and ZP3 have been inserted into mouse lines in which mouse ZP2 and ZP3 have been removed. Inserting the human $\mathrm{ZP} 2$ or $\mathrm{ZP} 3$ genes restores fertility with mouse but not human sperm (Rankin et al., $1998,2003)$. These results suggest that positive selection does not produce species-specific ZP3 or ZP2 sperm binding. However, analysis of the fate of ZP2 suggests a different possibility. In normal fertilization, sperm attachment cleaves ZP2, leading to reduced binding of later sperm (Rankin et al., 2003). Eggs expressing human $\mathrm{ZP} 2$ bind to mouse sperm, and allow fertilization, but ZP2 is not cleaved, and later sperm retain the ability to bind to fertilized eggs (Rankin et al., 2003). Thus the function of ZP2 may involve blocks to multiple sperm binding and prevention of polyspermy.

Evolutionary theory has suggested several possible mechanisms for these evolutionary patterns, including direction selection for sperm competitive ability, sexual selection, male-female conflict and reinforcement $(\mathrm{Wu}$, 1986; Gavrilets and Waxman, 2002; Gavrilets and Hayashi, 2005). These possibilities have been explored with fertilization experiments, analysis of coalescence patterns, models and comparison of molecular gene sequences within and between species. In most cases, the conclusion has been that rapid evolution of sperm loci probably requires rapid evolution of egg loci to drive it, and that the ultimate explanation of patterns of gamete recognition evolution requires joint understanding of the interaction of evolutionary forces in both males and females.

\section{Directional selection for fertilization efficiency}

An allele at a sperm locus that conferred an advantage during sperm competition by speeding the entry of a sperm into an egg might sweep through a population, becoming quickly fixed. If two species each separately accumulated these changes, then the resulting phylogenetic patterns might closely resemble the situation seen in abalone: high Dn/Ds ratios and low polymorphism. Because other gene sequences in abalone are highly polymorphic, low lysin polymorphism in a species implies a recent selective sweep at the lysin locus (Metz et al., 1998). The evolutionary model suggested by these data invokes a series of amino-acid mutations that start as polymorphisms but then sweep through the population due to directional selection on fertilization ability, resulting in a series of fixed amino-acid difference from the earlier sequence. Metz et al. (1998) estimated that a selective sweep would need to occur about once every few hundred thousand years to explain the rapid rate of lysin evolution.

Rapid sperm recognition evolution also can explain a common phylogenetic pattern among gamete recognition loci-rapid coalescence with species to generate reciprocal monophyly. For example, in the closely related Hawaiian sea urchins E. oblonga and E. mathaei, alleles at most nuclear loci share clades across species, but bindin is reciprocally monophyletic (Figure 2). Similarly, 
a Bindin is reciprocally monophyletic between species

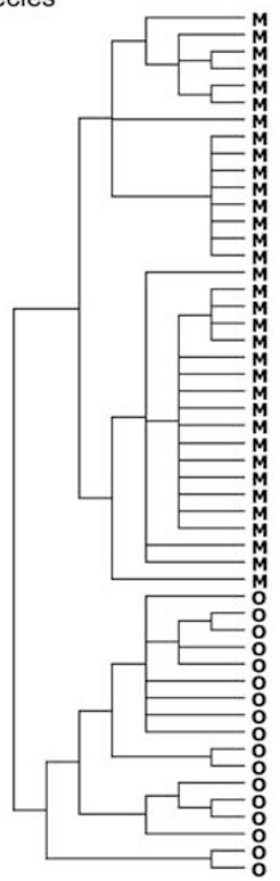

b tRNA-deacyclase is highly polyphyletic

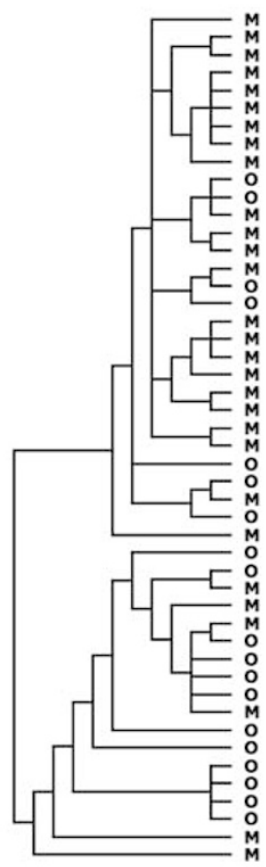

Figure 2 (a) The Hawaiian sea urchins Echinometra oblonga and E. mathaei are reciprocally monophyletic at bindin alleles despite a large amount of intraspecific polymorphism at this locus. (b) Allele genealogies at other loci including tRNA-deacyclase (shown here) show highly polyphyletic allele genealogies. Labels denote E. mathaei (M) or E. oblonga (O). Trees are from Heuristic searches in PAUP 4.0. (Data from Palumbi, 1999; SR Palumbi and J Alipaz, unpublished.)

so-called speciation genes in Drosophila show reciprocal monophyly whereas other genes do not, and show higher rates of amino-acid evolution than other loci (Tsaur and Wu, 1997; Ting et al., 2000). Monophyly produced by more rapid gene coalescence of gamete recognition loci is consistent with directional selection within species, though other evolutionary mechanisms may also generate this pattern (see below).

\section{What drives recurrent directional selection?}

Directional selection for sperm function is undoubtably a powerful force. However, sperm entry can probably not keep evolving to be faster and faster forever, and at some point directional selection is expected to be incapable of producing a faster sperm. Only if the egg receptors continue to change will the process of directional selection continue to operate.

Two types of mechanisms have been proposed to account for rapid change in receptor loci, which can then in principle drive rapid sperm recognition evolution. One mechanism depends on typical protein architecture of receptor proteins on egg surfaces. The other depends on conflicting selection in different sexes.

\section{Concerted evolution of repeated motifs}

In both sea urchins and abalone, the sperm receptor on the egg surface has been identified. Although the genes show no homology, they have several architectural features in common. The abalone receptor, VERL,

contains 22 tandemly repeated amino-acid motifs that each can bind to lysin (Figure 3). Similarly, the receptor on sea urchins eggs that attaches to bindin shows strong repetitive structure, including a region of hyalin-like repeats and a region made up of an alternating series of amino-acid domains termed TSP and CUB domains (Figure 3). The zona pellucida proteins that occur on mammalian egg surfaces and participate in gamete recognition are also made with repeated amino-acid motifs that permit self-assembly of $\mathrm{ZP}$ proteins into the egg surface (Jovine et al., 2005).

Positive selection among repeats of the abalone receptor has been reported at particular amino-acid positions in the first two repeats (Galindo et al., 2002). Repeats 3-22 are quite different in amino-acid sequence than repeats 1 and 2 and show very little amino-acid or nucleotide substitution among them. This led Swanson and Vacquier (1998) to suggest that concerted evolution among repeats was strong in this region. Their model suggests that concerted evolution shifts the frequency of repeats within a gene, and within a population, faster than would be possible by mutation alone, and that the lysin gene must evolve quickly at the amino-acid level to keep up.

However, this mechanism has limited explanatory power because the situation is very different in other taxa such as sea urchins. Here, concerted evolution does not homogenize the repeat array as it does in abalone. We have amplified sperm receptor repeats and extensively cloned them to estimate the number of different repeats in two individual Strongylocentrotus purpuratus (Figure 4). We found 13 and 14 different repeats in these two individuals (allowing for 1 or 2 base substitutions due to possible Taq and cloning error) in 26 and 27 clones, respectively (SR Palumbi and J Alipaz, in preparation). This suggests an average of 6-7 different repeats in the 11 repeat array in an individual allele, similar to the number of different repeats in the single cloned allele presented by Kamei and Glabe (2003). Other sea urchin species show similar patterns. In the closely related E. mathaei and E. oblonga, repeats are highly variable, and are not more similar within species than between species (Figure 4).

One possible explanation for this difference in concerted evolution between abalone and sea urchin receptors is that there are no introns within the abalone repeats (Galindo et al., 2002) whereas the urchin repeats each contain an intron of 200-400 bp (SR Palumbi and J Alipaz, in preparation). Lack of introns may favor unequal crossing over among very similar, adjacent repeats in abalone whereas divergence among introns in the sea urchin gene may slow down this process. Understanding the dynamics of concerted evolution awaits more information on the genomic sequence of sea urchin sperm receptors.

\section{Sexual conflict}

A different mechanism for evolution of receptors on eggs is the possibility that female and male components of the fertilization system are not mutually coevolved for the most efficient fertilization, but are evolving with conflicting priorities. In many species of free-spawning invertebrates, adult densities are high and sperm concentrations during spawning may be thick. This is 

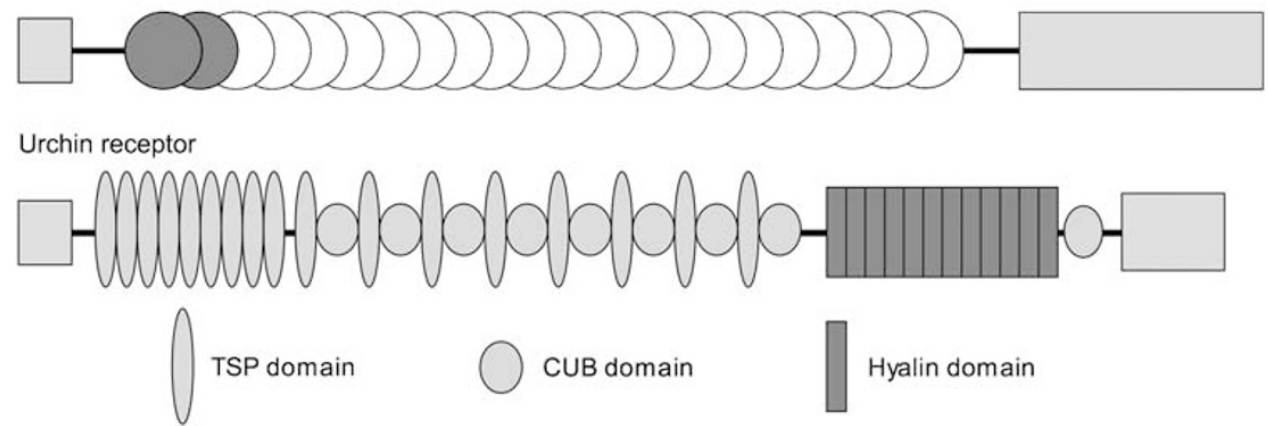

Figure 3 Architecture of sperm receptor proteins in abalone and sea urchins typically includes repeated amino-acid motifs. Motifs that bind sperm proteins are circles in the abalone receptor and rectangles for sea urchins. Motifs under positive selection have dark shading. The sea urchin receptor is modeled after Strongylocentrotus purpuratus. The congener S. franciscanus is reported to have a different arrangement of sperm binding motifs. (Modified from Galindo et al., 2002; Kamei and Glabe, 2003.)

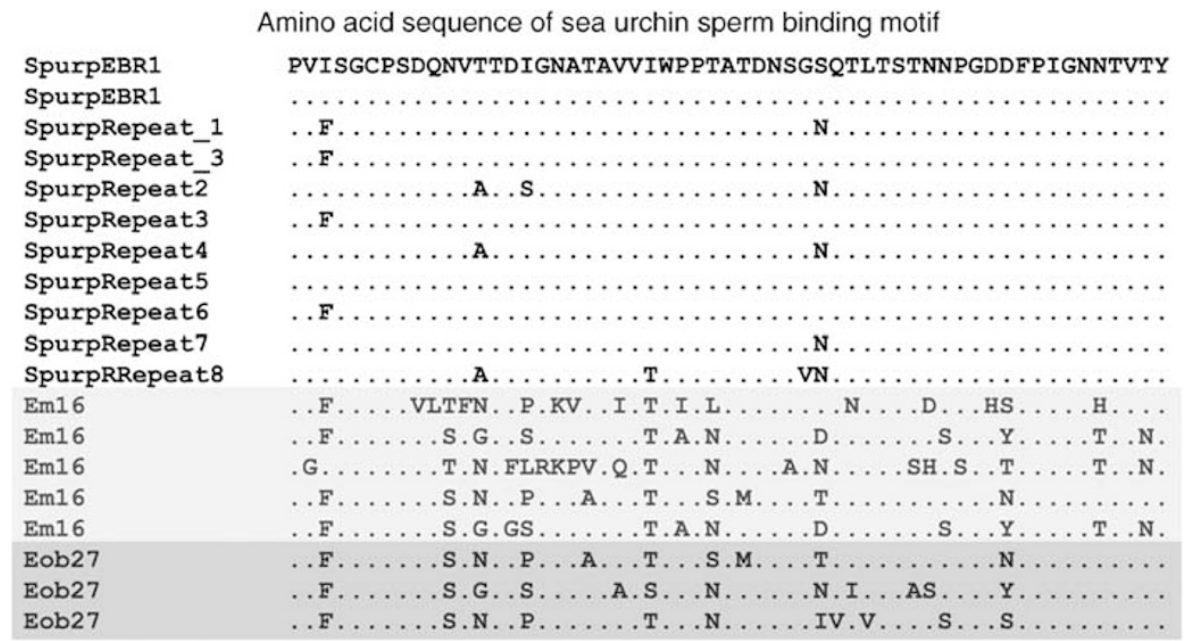

Figure 4 Amino-acid variation in the hyalin-like repeats motifs of the sea urchin sperm receptor within and between species. The top 11 sequences are from different repeats of the EBR1 gene sequenced from Strongylocentrotus purpuratus (Kamei and Glabe, 2003), showing variation at six amino-acid positions. Sequences from one individual Echinometra mathaei (Em16) and one E. oblonga (Eo27) show strong homology to S. purpuratus at about half of the amino-acid positions but are highly variable among repeats. Sequences were obtained by amplifying genomic DNA with primers that recognize intron-exon junctions present in each repeat, cloning PCR products into plasmid vectors and sequencing individual clones. (Data from Kamei and Glabe, 2003; SR Palumbi and J Alipaz, unpublished.)

probably true for the shallow water sea urchins, abalone, mussels and snails that have produced most of the gamete recognition patterns above (Levitan et al., 1992, 2007). In such circumstances, multiple sperm entry into eggs-polyspermy-can kill developing embryos (Levitan and Ferrell, 2006). When polyspermy is potentially high, male and female gametes have different priorities. Sperm will continue to be selected for fast entry into eggs because of directional selection for sperm competitive ability. However, eggs will also be under selection to evolve rapid barriers to polyspermy and to slow sperm entry.

Evidence of selection for barriers is common: sea urchins, for example, show an electrical block to polyspermy that acts within seconds of the entry of a sperm to prevent further sperm penetration (Metz et al., 1994). Evidence for mechanisms that slow sperm entry is more difficult to gather, but reproductive biologists have long known that it takes a large number of sperm to fertilize some eggs. In sea urchins, for example, fertilization rates are low unless there are hundreds to thousands of sperm per egg (Levitan et al., 1991), and the mathematic kinetics of sea urchin fertilization works best under the assumption that only $1 \%$ of the egg surface is actually fertilizable (Vogel et al., 1982).

Under polyspermic conditions, an allele at an egg surface protein locus might be favored that slowed sperm entry. Such an allele would increase in abundance, creating evolutionary pressure on sperm proteins to evolve to be more efficient at entering eggs that carry this allele. The cycle could continue, possibly producing round after round of directional selection on egg and sperm. Experiments on assortative mating based on egg and sperm genotypes showed that this mechanism was plausible (Palumbi, 1999). But this mechanism requires a continuous generation of novel alleles of the receptor, new mutation of the sperm genes to match them and replacement of the old alleles by the new.

These mechanisms also make a prediction that appears to be met in abalone but not sea urchins. The serial selective sweeps generated by the sexual conflict model detailed above should generate rapid coalescence and low polymorphism within species. This is the pattern seen in abalone (Metz et al., 1998). However, in sea 
urchins, bindin genes show a very different pattern, with a large amount of polymorphism within species as well as large differences between them. For example, in the Hawaiian sea urchin E. mathaei, there are at least five major clades of bindin alleles (Figure 2; see also Palumbi, 1999). Controlled crosses in which males homozygous for bindin clades A and B compete for access to eggs show that these different alleles confer different fertilization abilities (Palumbi, 1999). Thus, these bindin allele clades are not selectively neutral. To date, most sea urchin species that show positive selection of the bindin protein also show extensive polymorphism within species (Palumbi and Lessios, 2005).

The existence of many functionally different allele clades within a population is not predicted in a model in which strong directional selection drives the evolution of recognition proteins. However, experiments by Levitan and Ferrell (2006) showed in greater detail how conflict and coevolution might be happening and suggest that frequency dependent selection may drive gamete recognition coevolution in sea urchins.

\section{Rare allele advantage and gamete recognition}

Levitan and Ferrell (2006) set up spawning trials in field conditions, varying the number of males spawning and thereby varying the intensity of polyspermy. When polyspermy was low, males and females that had the most common bindin genotypes obtained the highest reproductive success (Levitan and Ferrell, 2006), whereas at higher male densities, males and females with unmatched bindin alleles produced the most larvae (Figure 5). This suggests that common alleles of bindin promote rapid sperm fusion, and that high densities of sperm overwhelm the egg's defenses against polyspermy. Eggs that possess rare alleles might slow down entry by sperm with dominant bindin alleles. At low sperm densities the most common alleles would fare best because they promote rapid fertilization. At high sperm

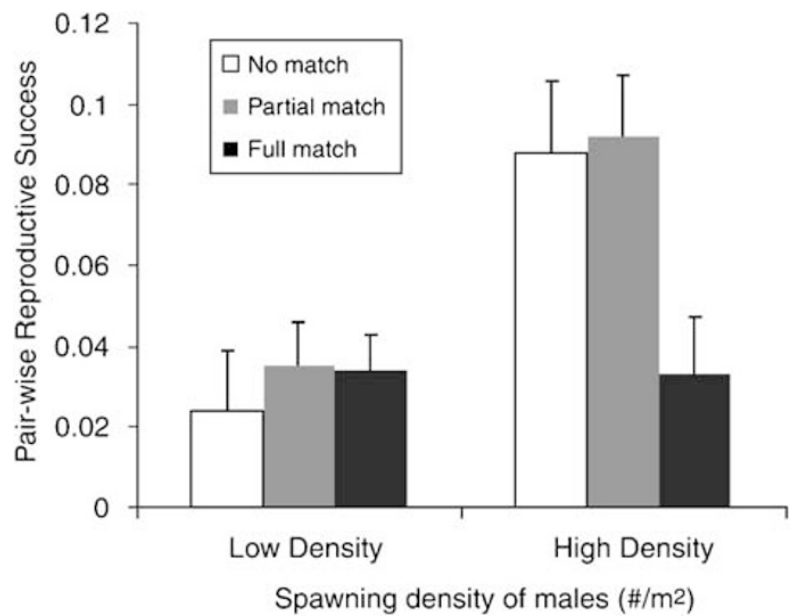

Figure 5 Relative reproductive success of male and female pairs that are a full match at bindin alleles (black bars), share no alleles (white bars) and have a partial match (shaded bars). When sperm concentrations are low, a full or partial match is slightly advantageous. When sperm concentration are high enough for polyspermy to be a significant source of larval mortality, fully matched parents have lower fitness than partially matches or nonmatched. (Data from Levitan and Ferrell, 2006.) densities eggs with rare alleles would have higher fitness because they suffer polyspermy less. These serial steps in the process of gamete coevolution have yet to be experimentally verified but current empirical observations suggest that these are likely.

Under conditions of high sperm density, rare egg alleles would be expected to spread, become common, cause the spread of bindin alleles that match them, and then suffer the disadvantages of the previously dominant egg and bindin alleles. This 'frontrunner' disadvantage, where the previous evolutionary leader becomes less fit because of its prominence in the population, creates a situation in which rare alleles have a higher relative fitness than common ones, and is a classic case of frequency dependent selection due to rare allele advantage and the disadvantage of common alleles.

Rare allele advantage generates balancing selection that slows allele extinction. As a result, this mechanism should allow for the persistence of allele clades older than those predicted by neutral coalescence models (Takahata, 1990), perhaps resulting in trans-species allele clades (Ioerger et al., 1990; Takahata, 1990; Richman et al., 1996; Hedrick, 1999). Thus loci dominated by balancing selection should coalesce within species more slowly than neutral loci (Takahata, 1990), not more quickly as bindin.

Bindin thus shows two different evolutionary patterns that are difficult to reconcile. Positive selection between species and rapid coalescence suggest the action of direction selection and selective sweeps. High levels of functional polymorphism within species and the persistence of very different alleles suggest rare allele advantage or some other form of balancing selection. How directional and balancing selection could act in concert to produce functional divergence as well as polymorphism, or whether a different evolutionary mechanism could be operating, is an important goal for future research.

\section{Other forms of balancing selection based on gamete architecture}

Frequency-dependent selection can promote polymorphism by slowing the rate of extinction of rare alleles and it can generate positive selection among alleles within species, so the discovery of rare allele advantage in this system is an important step in understanding the conundrum of sea urchin bindin variation. However, other mechanisms of balancing selection might also play this role and could be operating in this and other gamete recognition systems. The way gamete recognition genes are expressed and the way these multi-locus products interact suggests an additional, general mechanism of balancing selection based on heterozygote superiority.

The nature of sperm attachment and sperm receptor proteins is that they are expressed abundantly on the surfaces of sperm and eggs, respectively. Their interaction is not the interaction of single protein molecules in isolation but is instead the interaction of proteins on surfaces. In the case of heterozygotes, these surfaces will be covered with proteins that, if they derive from different alleles, might be functionally heterogeneous. What alleles are expressed by haploid gametes?

Expression of most genes in gametes occurs at the stages preceding meiosis, and so the expectation is that 
both parent's alleles are probably expressed equally, however few data exist on the expression of gamete recognition proteins. We tested the assumption of diploid expression by backcrossing field-collected hybrid $E$. mathaei $\times E$. oblonga males with females from each species and scoring the resulting larvae for bindin genotype. If each sperm produces only the bindin protein from the allele it carries, then only hybrid sperm with a mathaei allele will fertilize mathaei eggs, and only sperm with an oblonga allele will fertilize oblonga eggs: in both cases, all backcrossed larvae will be homozygous for bindin. In fact, $50 \%$ of backcrossed larvae were heterozygous for bindin alleles, exactly the prediction made if all hybrid sperm carry both alleles and can fertilize eggs of both species equally (SR Palumbi (unpublished); $n=5$ crosses, average larvae scored per cross $=25$ ). These data suggest, at least for sea urchin bindin, that individual sperm express both alleles and carry both bindin allotypes.

\section{Evolutionary consequences of dual allele expression: polymer selection}

For any locus, new mutant alleles occur, at first, strictly in heterozygotes. When gametes express both recognition alleles carried by the adult that made them, this produces a densely packed heteropolymer on gamete surfaces. Imagine a population monomorphic for bindin allele S. If $S$ mutates to $S^{\prime}$ in a male, it will appear as a $S S^{\prime}$ heterozygote producing a $S^{\prime}$ sperm surface. Should the $\mathrm{SS}^{\prime}$ surface interact less well with an egg (for example, if SS' fertilizes less well than SS) then the $S^{\prime}$ allele is likely to be lost. If $S$ and $S^{\prime}$ proteins function better when they are together in heteropolymers, $S^{\prime}$ will spread in the population. This argument could apply to any locussuperior heterozygotes will always be selected for. A key difference between this model and standard models of protein evolution is that the initial selection is for alleles that function well together on heterogeneous surfaces where gamete binding requires the joint action of many protein molecules acting in concert. Thus an SS ${ }^{\prime}$ heterozygote is expected to perform better than SS homozygotes, but may also perform better than $S^{\prime} S^{\prime}$ homozygotes. Even if the relative fitness of $S^{\prime} S^{\prime}$ is less than SS, the superiority of SS' maintains both alleles in the population. The same can be true for other loci-for example, the locus that leads to cystic fibrosis in humans (Palumbi, 2001)_but for proteins that function strictly on their own, such as monomeric enzymes for instance, selection for joint beneficial function of two different alleles may not occur often.

This basic mechanism suggests that new, functionally different alleles will be selected and spread quickly if they make superior heterozygotes. The same is true of sperm receptors on egg surfaces, though here sexual conflict may select for $\mathrm{EE}^{\prime}$ heterozygotes that fertilize less well under high sperm concentrations. Alternatively, directional selection might lead to better overall fertilization if sperm are rare. If this model reflects ongoing evolutionary forces, they should generate heterozygote superiority among at least some gamete recognition alleles.

\section{Can polymer selection cause heterozygote superiority?}

The above verbal model suggests that common, closely related alleles of bindin should show heterozygote superiority in fertilization rate. Heterozygote superiority is known to be associated with both positive selection and high polymorphism rates in MHC (Hughes and Nei, 1989; Takahata et al., 1992; Hedrick, 1999; Sauermann et al., 2001). Positive selection among MHC alleles generates diversity in a set of critically important amino-acid positions important in self-/non-self-recognition. A similar process, in principle, might act at bindin positions (for example, the 'hot spot' described in Metz and Palumbi, 1996) that function in proteins on the egg surface. If true, then a common bindin allele might mutate to form a derived allele with which it works well in heteropolymers. Do such superior heterozygotes exist in natural populations?

As a first step to testing for heterozygote superiority in bindin, we performed a series of sperm competition experiments with male E. mathaei sea urchins heterozygous for the most common bindin alleles A and B (see clade designations in Palumbi, 1999). When AA and AB sperm were mixed together and used to fertilize eggs from AA females, there were $70 \%$ more offspring produced by the heterozygous male than the homozygous male, after correcting for minor differences in sperm concentration (Figure 6). Polyspermy is not a strong factor in these experiments, run at moderate sperm concentrations, and the result is not likely to be due to death of multiply fertilized zygotes. Instead these preliminary studies suggest that AB males have a fertilization advantage over AA males.

If this pattern of heterozygote superiority were common among bindin alleles, it might add to the rare allele advantage caused by sexual conflict. In this case, successful alleles would not be defined by their action as homozygotes but by their action as heterozygotes. The evolutionary prediction based on sexual conflict would be that sister alleles of sperm attachment proteins would show better or faster fertilization when expressed in heterozygotes together, and that sister alleles of sperm receptors would show heterozyogotes with slower fertilization. The first prediction is met in the experiments described above (Figure 6). The complex nature of sperm receptor alleles in sea urchins, made up of many

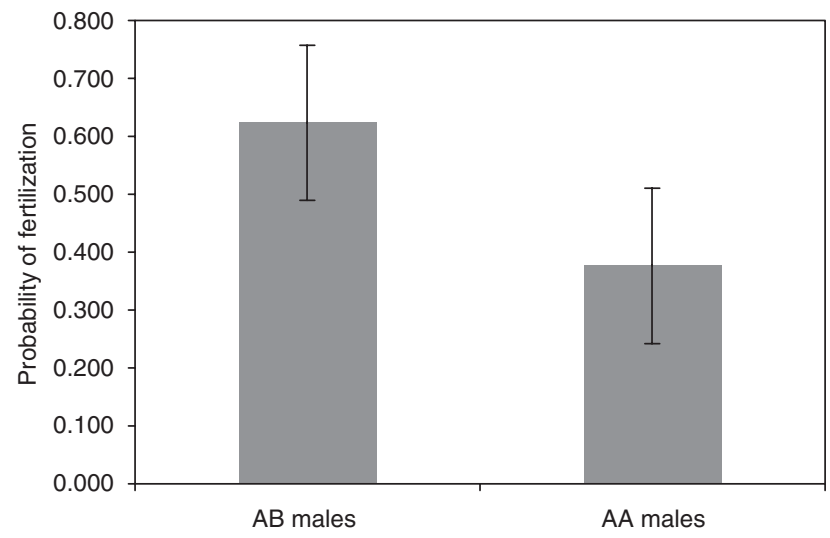

Figure 6 Relative probability of fertilization of sea urchins eggs by heterozygous Echinometra mathaei males with $\mathrm{AB}$ bindin genotypes compared to AA males. Shown are means and standard deviations across a total of 12 separate crosses, derived from eggs of five different females with AA bindin genotypes. Experiments using eight $\mathrm{AB}$ males and six $\mathrm{AA}$ males were conducted as described in Palumbi (1999). 
different repeats, makes the second prediction hard to test. In cases for male and female loci, polymorphism would be enhanced for alleles that work well together.

\section{Gamete recognition evolution and speciation}

Rapid evolution of gamete recognition genes has the potential to explain rapid marine speciation if this evolution can lead to more rapid gamete incompatibility between populations (Palumbi, 1992). Mechanisms that promote polymorphism and positive selection by themselves do not necessarily generate population or species divergence. Instead, population divergence most likely requires rounds of gamete coevolution in which different evolutionary mechanisms come into play:

- Sperm competition for rapid fertilization may lead to selective sweeps or adept heterozygotes that generate highly fertile sperm.

- This adaptation in the presence of high sperm densities could generate selection for new egg surface alleles that slow sperm entry.

- New sperm alleles might arise that restore fertilization speed, sparking...

- A new round of egg surface evolution, and so on.

Should these cycles generate new alleles within a population that do not occur elsewhere, then allopatric populations may diverge more quickly than they would under purely neutral drift. This is the essence of rapid speciation by evolution of gamete recognition.

\section{Is allopatry necessary?}

Recent models have questioned whether this scenario depends on allopatry or whether sympatric populations can also experience rapid reproductive divergence. Gavrilets and Waxman (2002) showed that female and male coevolution can result from the pressures generated by polyspermy, and that the result would often be diversification of female alleles into divergent fertilization classes. In some cases, male alleles would also diversify, forming two clusters of coadapted male and female alleles. Further evolution of these clusters could form reproductively isolated species. In other cases, males would be selected for adaptation to the middle of the egg adaptive landscape, not fully adapted to different female strategies, but 'trapped' in between them.

Van Doorn et al. (2001) showed that egg surface diversification would lead to sperm attachment diversification. If egg surface genes were independent of ecological resource competition, then a selective sweep might occur. If egg surface genes become correlated with ecological types, then different mating types specialize on different resources and reproductively isolated subpopulations could evolve (Van Doorn et al., 2001). In both models, a single population can evolve into two sympatric species, though the evolutionary forces driving these dynamics differ.

Empirical evidence supports some aspects of both sympatric models. Sexual conflict due to polyspermy drives the Gavrilets approach, and has been suggested in some but not all sea urchins (Levitan and Ferrell, 2006; Levitan et al., 2007). The Van Doorn model depends on the egg receptor reaching a critical threshold of egg surface diversity. Though early data from abalone suggested this might be minimal because of the homogenizing effects of concerted evolution (Swanson and Vacquier, 1998), data from sea urchins shows profligate variation (Figure 4; Kamei and Glabe, 2003). The Van Doorn model also presupposes some ecological diversification, a common feature of sympatric sea urchin and abalone species (Lessios, 2007).

\section{Reproductive character displacement}

The processes detailed above can act within or between populations to generate reproductive isolation de novo. However, other processes might act to push previously separated species to have more divergent gamete recognition systems. Reproductive character displacement occurs when species in sympatry show more reproductive divergence than when they live allopatrically. This phenomenon, termed reinforcement when it is driven by low hybrid fitness, may be rare in animals because it is likely to be strong only when species come into contact and commonly cross-fertilize to produce low fitness offspring.

In sea urchin genera that have many pairs of sympatric species, bindin shows a high rate of evolution and a high Dn/Ds ratio (Metz et al., 1998; Palumbi and Lessios, 2005; Lessios, 2007). This pattern could be due to more rapid sympatric speciation in taxa with high rates of bindin evolution. However, it could also be due to reinforcement in which bindin evolution is accelerated in situations with coexisting, closely related species. A third alternative exists: that only species with high rates of bindin evolution and subsequent gamete incompatibility can coexist sympatrically after allopatric speciation and range expansion.

More direct evidence for reproductive character displacement comes from comparison of a widely ranging species in the Pacific, E. oblonga. In areas where it lives in sympatry with a closely related (as yet unnamed) species Echinometra species C, the bindin alleles of E. oblonga are divergent from those of allopatric E. oblonga populations, and show a signal of positive selection (Geyer and Palumbi, 2003). The combination of divergence in sympatry and positive selection suggests the action of reinforcement, which might be acting to fix mutations in gamete recognition loci among coexisting species that can prevent fertilization.

Reinforcement in this system is most likely acts first on females, causing egg surface proteins to diverge to prevent an egg from being fertilized by the wrong sperm. Such selection against gamete wastage is not as likely to act on males that produce billions of sperm. As a consequence, reinforcement should be strongest on egg surface proteins: bindin would then evolve in response to these changes. A prediction of this model would be that sympatric E. oblonga would have more divergent receptor repeats than allopatric E. oblonga, but this hypothesis has not been tested.

\section{The importance of ecology}

External fertilization systems are particularly sensitive to the density of spawning adults, largely because fertilization success is an increasing function of sperm density. Though the impact of adult density on fertilization ecology has long been appreciated (for example, Levitan 

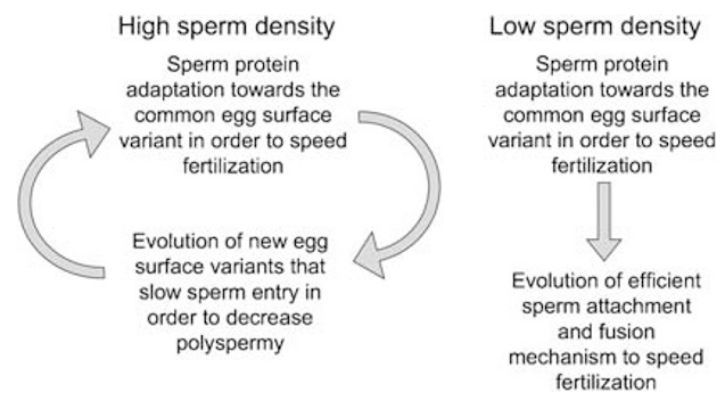

Figure 7 Cyclic and directional modes of gamete adaptation (left and right, respectively) that derive from ecological conditions favoring high sperm and low sperm densities.

et al., 1992), the impact of adult density on patterns of gamete recognition evolution has only recently been explored (Levitan and Ferrell, 2006).

In particular, the cyclic selection model of gamete evolution (Figure 7, left), driven by a race between egg surface proteins and sperm attachment proteins, depends on the importance of polyspermy. When adult densities are low, polyspermy is rare, and any disadvantage of common bindin alleles that promotes rapid fertilization might fade. In such cases, fertilization evolution might flip to a directional mode in which adaptation of both male and female components maximizes fertilization success (Figure 7, right).

Low male density can occur for a large number of ecological reasons. Deep-sea species on abyssal plains often have low adult densities because of lower food supplies, and may seldom occur in densities high enough to saturate eggs with sperm. The only deep sea species for which gamete recognition genes are known is Allocentrotus fragilis, a close relative of $S$. purpuratus and S. droebachiensis: its bindin is less divergent from the common ancestor of these three species than is the bindin of the other two, shallower water species (Biermann, 1998). Rates of evolution of sperm attachment proteins in other deep-sea taxa with low population densities would provide an interesting comparison to shallow water species.

Low densities in shallow water species can also occur, due to factors such as food limitation, recent introduction, rarity of refuges or abundance of predators. Because these ecological factors can vary over time, a species might find itself in both cyclic and directional modes of gamete evolution at different times.

One common ecological situation in which population densities might change is when a species invades a new habitat. At first the invader is likely to be uncommon, favoring evolution for enhanced fertilization efficiency or, if there is a common congener present, enhanced conspecific sperm precedence. Later, as population densities increase, the species may enter a cyclic evolutionary phase in which selection on eggs to resist polyspermy is enhanced. Species that persist in metapopulations, in which local demes experience periodic extinction and recolonization, may typically shift between directional and cyclic gamete adaptation. Whether a long period of cyclic adaptation decreases fertilization ability enough to slow the ability of such species to invade a new habitat is unknown.

\section{Conclusions and summary}

Interactions of gamete recognition genes expressed on eggs and sperm represent a simple genetic system to understand the evolutionary forces that act on reproductive isolation. Yet, the patterns shown by these genes and the processes that act on them are complex reflections of mating system interactions and population ecology. Gamete recognition evolution is frequently characterized by rapid coalescence within species, positive selection, selective sweeps or rampant polymorphism of functionally different alleles. A key feature of scenarios that explain gamete evolution is that sperm proteins are expected to adapt to proteins on the egg surface. As a result, rapid or consistent evolution of sperm genes is likely to require rapid or consistent evolution of egg surfaces. Mechanisms to explain egg surface evolution are complicated by the repeat structure of these proteins and the possibility that additional forces such as concerted evolution act on them. Egg surfaces might coevolve with sperm in two fundamentally different ways. The first is directional selection based on coevolution to cooperatively tune the fertilization system to be as efficient as possible. The second is cyclic adaptation based on coevolution of sperm to increase fertilization followed by evolution of eggs to decrease fertilization efficiency and thereby reduce polyspermy, a form of sexual conflict.

Empirical evidence for these mechanisms includes allele-specific function of sperm proteins, and decreased fitness of common sperm alleles under polyspermic conditions. Evidence for rare allele advantage and heterozygote superiority both provide examples of how particular alleles can affect gamete function and play a role in reproductive success.

Independent coevolution of egg and sperm proteins in allopatric populations could generate rapid protein divergence that in turn could be responsible for low cross-fertilization potential between these populations. Reproductive isolation generated this way might be enhanced by reinforcement when previously allopatric populations came back together. Mathematical models show that both sexual conflict and association of gamete recognition genes with ecological differentiation can drive the diversification of a population into two reproductively isolated populations. Allopatry is not required for these models to function.

Most likely, coevolutionary mechanisms that act within populations, such as sexual selection or sexual conflict are critical to patterns of gamete evolution and polymorphism. Forces that act between allopatric populations or between species to enhance genetic divergence at these loci may also play a role.

\section{References}

Biermann C (1998). The molecular evolution of sperm bindin in six species of sea urhcins (Echinoida: Strongylocentrotidae). Mol Biol Evol 15: 1761-1771.

Biermann CH, Marks JA, Vilela-Silva A, Castro MO, Mourao PAS (2004). Carbohydrate-based species recognition in sea urchin fertilization: another avenue for speciation? Evol Dev 6: 353-361.

Coyne JA, Orr HA (2004). Speciation. Sinauer Press: Sunderland, MA, 545pp. 
Davis JC, Brandman O, Petrov DA (2005). Protein evolution in the context of Drosophila development. J Mol Evol 60: 774-785.

Debenham P, Brzezinski MA, Foltz KR (2000). Evaluation of sequence variation and selection in the bindin locus of the red sea urchin, Strongylocentrotus franciscanus. J Mol Evol 51: 481-490.

Eberhard W (1996). Female Control: Sexual Selection by Cryptic Female Choice. Princeton University Press: Princeton, NJ.

Galindo BE, Moy GW, Swanson WJ, Vacquier VD (2002). Fulllength sequence of VERL, the egg vitelline envelope receptor for abalone sperm lysin. Gene 288: 111-117.

Galindo BE, Vacquier VD, Swanson WJ (2003). Positive selection in the egg receptor for abalone sperm lysin. Proc Natl Acad Sci USA 100: 4639-4643.

Gavrilets S, Hayashi TI (2005). Speciation and sexual conflict. Evol Ecol 19: 167-198.

Gavrilets S, Waxman D (2002). Sympatric speciation by sexual conflict. Proc Natl Acad Sci USA 99: 10533-10538.

Geyer LB, Palumbi SR (2003). Reproductive character displacement and the genetics of gamete recognition in tropical sea urchins. Evolution 57: 1049-1060.

Geyer LB, Palumbi SR (2005). Conspecific sperm precedence in two species of tropical sea urchins. Evolution 59: 97-105.

Hedrick PW (1999). Balancing selection and MHC. Genetics 104: 207-214.

Hellberg ME, Moy GW, Vacquier VD (2000). Positive selection and propeptide repeats promote rapid interspecific divergence of a gastropod sperm protein. Mol Biol Evol 17: $458-466$

Howard D (1999). Conspecific sperm and pollen precedence and speciation. Annu Rev Ecol Syst 30: 109.

Howard DJ, Palumbi SR, Birge L, Manier MK (2008). Sperm and speciation. In: Birkhead T, Hosken D, Pitnick S (eds). Sperm Biology: An Evolutionary Perspective. Elsevier Press: NY.

Hughes AL, Nei M (1988). Pattern of nucleotide substitution at major histocompatibility complex loci reveals overdominant selection. Nature 335: 167-170.

Hughes AL, Nei M (1989). Nucleotide substitution at major histocompatibility complex class II loci: evidence for overdominant selection. Proc Natl Acad Sci USA 86: 958-962.

Ioerger IR, Clarke AG, Kao T-H (1990). Polymorphism at the self-incompatibility locus in Solanaceae predates speciation. Proc Natl Acad Sci USA 87: 9732-9735.

Jovine L, Darie CC, Litscher ES, Wassarman PM (2005). Zona pellucida domain proteins. Annu Rev Biochem 74: 83-114.

Kamei N, Glabe CG (2003). The species-specific egg receptor for sea urchin sperm adhesion is EBR1, a novel ADAMTS protein. Genes Dev 17: 2502-2507.

Lee YH, Ota T, Vacquier VD (1995). Positive selection is a general phenomenon in the evolution of abalone sperm lysin. Mol Biol Evol 12: 231-238.

Lessios HA (2007). Reproductive isolation between species of sea urchins. Bull Mar Sci 81: 191-208.

Levitan DR, Ferrell DL (2006). Selection on gamete recognition proteins depends on sex, density, and genotype frequency. Science 312: 267-269.

Levitan DR, Sewell MA, Chia F-S (1991). Kinetics of fertilization in the sea urchin Srongylocentrotus franciscanus: interaction of gamete dilution, age, and contact time. Biol Bull 181: 371-378.

Levitan DR, Sewell MA, Chia F-S (1992). How distribution and abundance influence fertilization success in the sea urchin Strongylocentrotus franciscanus. Ecology 73: 248-254.

Levitan DR, terHorst CP, Fogarty ND (2007). The risk of polyspermy in three congeneric sea urchins and its implications for gametic incompatibility and reproductive isolation. Evolution 61: 2007-2014.

Metz EC, Kane RE, Yanagimachi H, Palumbi SR (1994). Specificity of gamete binding and early stages of fusion in closely related sea urchins (genus Echinometra). Biol Bull 187: 23-34.

Metz EC, Palumbi SR (1996). Positive selection and sequence rearrangements generate extensive polymorphism in the gamete recognition protein bindin. Mol Biol Evol 13: 397-406.

Metz EC, Robles SR, Vacquier VD (1998). Nonsynonymous substitution in abalone sperm fertilization genes exceeds substitution in introns and mitochondrial DNA. Proc Natl Acad Sci USA 95: 10676-10681.

Palumbi SR (1992). Marine speciation on a small planet. Trends Ecol Evol 7: 114-118.

Palumbi SR (1999). All males are not created equal: fertility differences depend on gamete recognition polymorphisms in sea urchins. PNAS 96: 12632-12637.

Palumbi SR (2001). Humans as the world's greatest evolutionary force. Science 293: 1786-1790.

Palumbi SR, Lessios HA (2005). Evolutionary animation: how do molecular phylogenies compare to Mayr's reconstruction of speciation patterns in the sea? Proc Natl Acad Sci USA 102: $6566-6572$.

Palumbi SR, Metz E (1991). Strong reproductive isolation between closely related tropical sea urchins (genus Echinometra). Mol Biol Evol 8: 227-239.

Panhuis TM, Clark NL, Swanson WJ (2006). Rapid evolution of reproductive proteins in abalone and Drosophila. Philos Trans $R$ Soc Lond B Biol Sci 361: 261-268.

Rankin TL, Coleman JS, Epifano O, Hoodbhoy T, Turner SG, Castle PE et al. (2003). Fertility and taxon-specific sperm binding persist after replacement of mouse sperm receptors with human homologs. Dev Cell 5: 33-43.

Rankin TL, Tong ZB, Castle PE, Lee E, Gore-Langton R, Nelson LM et al. (1998). Human ZP3 restores fertility in Zp3 null mice without affecting order-specific sperm binding. Development 125: 2415-2424.

Richman AD, Uyenoyama M, Kohn JR (1996). Allele diversity and gene genealogy at the self-incompatibility locus in the Solanaceae. Science 273: 1212-1216.

Riginos C, Wang D, Abrams AJ (2006). Geographic variation and positive selection on M7 lysin, an acrosomal sperm protein in mussels (Mytilus spp.). Mol Biol Evol 23: 1952-1965.

Sauermann U, Nürnberg P, Bercovitch FB, Berard JD, Trefilov A Widdig A et al. (2001). Increased reproductive success of MHC class II heterozygous males among free-ranging rhesus macaques. Hum Genet 108: 249-254.

Servedio MR, Noor MAF (2003). The role of reinforcement in speciation: theory and data. Ann Rev Ecol Evol Syst 34: 339-364.

Swanson W, Vacquier VD (1998). Concerted evolution in an egg receptor for a rapidly evolving abalone sperm protein. Science 281: 710-712.

Swanson WJ, Vacquier VD (2002a). Reproductive protein evolution. Annu Rev Ecol Syst 33: 161-179.

Swanson WJ, Vacquier VD (2002b). The rapid evolution of reproductive proteins. Nat Rev Genet 3: 137-144.

Swanson WJ, Zhang ZH, Wolfner MF, Aquadro CF (2001). Positive Darwinian selection drives the evolution of several female reproductive proteins in mammals. Proc Natl Acad Sci USA 98: 2509-2514.

Takahata N (1990). A simple genealogical structure of strongly balanced allelic lines and trans-species evolution of polymorphism. Proc Natl Acad Sci USA 87: 2419-2423.

Takahata N, Satta Y, Klein J (1992). Polymorphism and balancing selection at major histocompatibility complex loci. Genetics 130: 925-938.

Thomas S, Singh RS (1992). A comprehensive study of genic variation in natural-populations of Drosophila melanogaster. 7. Varying rates of genic divergence as revealed by 2-dimensional electrophoresis. Mol Biol Evol 9: 507-525.

Ting C-T, Tsaur S-C, Wu C-I (2000). The phylogeny of closely related species as revealed by the genealogy of a speciation gene, Odysseus. Proc Natl Acad Sci USA 97: 5313-5316. 
Tomaiuolo M, Hansen TF, Levitan DR (2007). A theoretical investigation of sympatric evolution of temporal reproductive isolation as illustrated by marine broadcast spawners. Evolution 61: 2584-2595.

Tsaur SC, Wu CI (1997). Positive selection and the molecular evolution of a gene of male reproduction, Acp26Aa of Drosophila. Mol Biol Evol 14: 544-549.

Turner LM, Hoekstra HE (2006). Adaptive evolution of fertilization proteins within a genus: variation in ZP2 and ZP3 in deer mice (Peromyscus). Mol Biol Evol 23: 1656-1669.

Vacquier VD, Carner KR, Stout CD (1990). Species specific sequences of abalone lysin, the sperm protein that creates a hole in the egg envelope. Proc Natl Acad Sci USA 87: 5792-5796.

Van Doorn GS, Luttikhuizen PC, Weissing FJ (2001). Sexual selection at the protein level drives the extraordinary divergence of sex-related genes during sympatric speciation. Proc R Soc Lond B Biol Sci 268: 2155-2161.

Vieira A, Miller DJ (2006). Gamete interaction: is it speciesspecific? Mol Reprod Dev 73: 1422-1429.

Vogel H, Czihak G, Chang P, Wolf W (1982). Fertilization kinetics of sea urchin eggs. Math Biosci 58: 189-216.

Willis BL, van Oppen MJH, Miller DJ, Vollmer SV, Ayre DJ (2006). The role of hybridization in the evolution of reef corals. Ann Rev Ecol Evol Syst 37: 489-517.

Wu C-I (1986). A stochastic simulation study on speciation by sexual selection. Evolution 39: 66-82.

Wyckoff GJ, Wang W, Wu C-I (2001). Rapid evolution of male reproductive genes in the descent of man. Nature 403: 304-309.

Zigler KS, McCartney MA, Levitan DR, Lessios HA (2005). Sea urchin bindin divergence predicts gamete compatibility. Evolution 59: 2399-2404. 